\title{
An Optimization Model for the Design of Fast Working Face
}

\author{
Yili SONG, Die HU, Qingtao LIU and Xue ZHANG ${ }^{1}$ \\ Xiangtan University, China
}

\begin{abstract}
Whether the parabola can be close to an ideal parabolic surface has an important effect on the working efficiency of FAST. In this paper, the influence mechanism of radial expansion of FAST actuator on the shape adjustment of active reflector is analyzed, so as to explore the scheme to determine the ideal working face of FAST. Based on the geometric relations of paraboloids, an optimal model for determining the ideal paraboloid was established, and then euler transformation was carried out on the coordinate positions of the celestial bodies, and the hierarchical iterative algorithm was used to adjust the reflection surface, and the optimal model for adjusting the working paraboloid was established. Finally, the MATLAB was used to solve the model to determine the best working face position of FAST, and calculated that the reception efficiency of the feed cabin after adjustment has been improved by more than $90 \%$. The optimization model established in this paper and the research results of layered iterative algorithm can be used as a reference for the working face adjustment of radio telescope in engineering application.
\end{abstract}

Keywords. FAST ideal paraboloid, optimal model, Euler transformation, hierarchical iterative algorithm.

\section{Introduction}

FAST consists of an active reflector, a feed cabin and its related control, measurement and support system.As shown in figure 1,

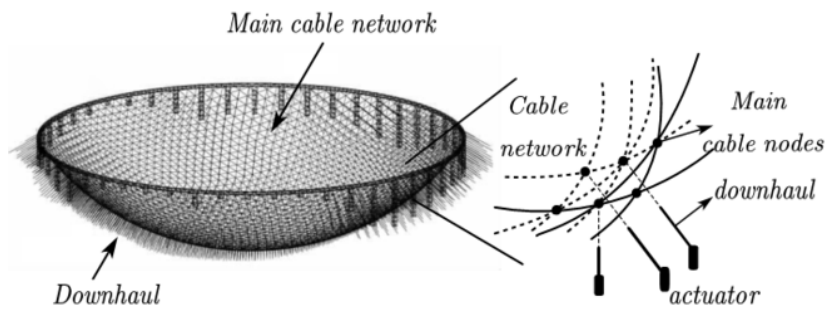

Figure 1. Schematic diagram of active reflector system

Active reflector can be divided into two states: working state and reference state. A spherical surface with a radius of 300 meters and a aperture of 500 meters; The operating state is the reference state is adjusted to an approximately rotating paraboloid with a

1 Corresponding Author, Xue ZHANG, Xiangtan University, China; Email: 201905555305@smail.xtu.edu.cn 
diameter of 300 meters. In working condition, concentric spheres with radius difference $\mathrm{F}=0.466 \mathrm{R}$ are formed between the center moving region of the feed cabin and the sphere where the datum plane is located. When FAST observes celestial body $\mathrm{S}$, the intersection point of the straight line SC formed by S and concentric spherical center $\mathrm{C}$ and the focal plane of the moving region of the feed capsule is the focus of the paraboloid in the working state of the active reflector.

In our research, we use the optimization model established by multiple constraints to solve the active reflection surface of the ideal paraboloid to determine, under multiple constraints using layered iterative algorithm for fitting operation, easy to calculate a relatively small amount of calculation results. Finally, the receiving efficiency of the feed cabin is calculated and the ideal data is obtained, which shows the feasibility and advantages of our model.

\section{Establishment of Ideal Paraboloid Determination Model}

\subsection{The Determination of Parabolic Equation}

The object $S$ is located directly above the reference sphere, that is, $\alpha=0^{\circ} \beta=90^{\circ}$, the center of the reference sphere as the origin point $C$, to measure the celestial body $S$ and the center of the spherical $C$. The sectional view of FAST in the established coordinate system is shown in figure 2 ,

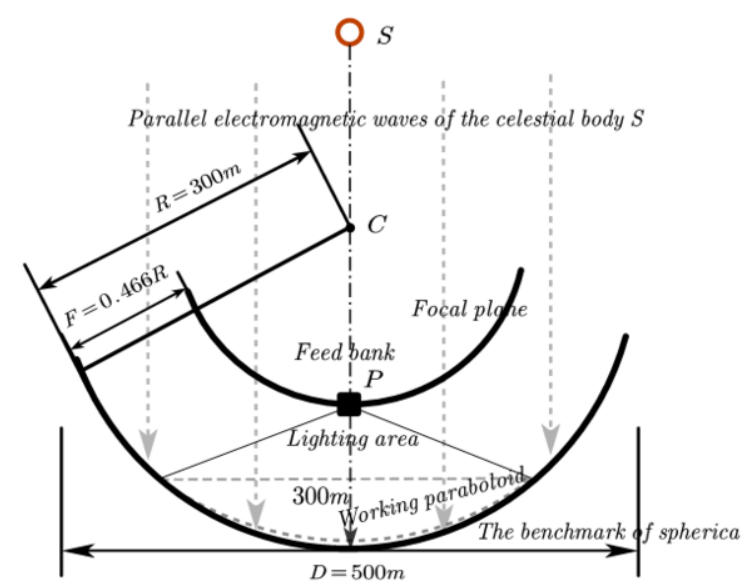

Figure 2. FAST section view when the object is located directly above the reference sphere

We assume that the coordinate at the bottom of the parabola is $A(0, a)$, then the equation of the parabola is,

$$
x^{2}=2 p(z-a)
$$

Where, $p$ represents focal length, and $a$ represents the distance between the base A and the origin $O$. And then we rotate the parabola 180 degrees around the axis $z$ to get the equation of the parabola in this case, 


$$
x^{2}+y^{2}=2 p(z-a)
$$

\subsection{Calculation of Main Cable Point Correlation Parameters}

\subsubsection{Equation of a Line Formed By the Principal Cable Point and the Spherical Center Point $\mathrm{O}$}

In the spatial rectangular coordinate system established in the previous process, the coordinates of the spherical center point are $(0,0,0)$, and the coordinates of the main cable $i$ point are $\left(x_{i}, y_{i}, z_{i}\right)$. According to the formula between two points in space,

$$
\frac{x-0}{x_{i}-0}=\frac{y-0}{y_{i}-0}=\frac{z-0}{z_{i}-0}
$$

2.2.2. The Distance Between the Main Cable Point and the Corresponding Point on the Ideal Parabola

It is known that the main cable point moves radially through the change of the actuator. According to the previous analysis, the solution formula of the ideal point is,

$$
\left\{\begin{array}{c}
\frac{x-0}{x_{i}-0}=\frac{y-0}{y_{i}-0}=\frac{z-0}{z_{i}-0} \\
x^{2}+y^{2}=2 p(z-a)
\end{array}\right.
$$

Then, the distance $d_{j}$ between each main cable point and the ideal point can be obtained from the distance formula,

$$
d_{j}=\sqrt{\left(x_{j}-x_{j}{ }^{\prime}\right)^{2}+\left(\mathrm{y}_{j}-\mathrm{y}_{j}{ }^{\prime}\right)^{2}+\left(\mathrm{z}_{j}-\mathrm{z}_{j}{ }^{\prime}\right)^{2}}
$$

among them, $j=1, \cdots, k, k+1$

Therefore, the critical point of the paraboloid can be obtained according to the section equation of the paraboloid and the sphere at the aperture as follows,

$$
\left\{\begin{array}{c}
x^{2}+y^{2}=150^{2} \\
x^{2}+y^{2}=2 p(z-a)
\end{array}\right.
$$

\subsection{A Deterministic Model of an Ideal Paraboloid}

First of all, make sure all of the $d_{i}$ is in the interval $(-0.6,0.6)$, and define,

$$
\begin{aligned}
& \operatorname{sgn}\left(d_{j}\right)=\left\{\begin{array}{l}
0, d_{j} \leqslant 0.6 \\
1, d_{j}>0.6
\end{array}\right. \\
& n=\sum_{i=1}^{k+1} \operatorname{sgn}\left(d_{j}\right)
\end{aligned}
$$

Here, $n$ represents the number of main cable points within the radial contraction range. 
In order to measure the fitting accuracy of the main cable net surface and the ideal paraboloid, we root $d_{i}$ mean square, that is $R M S(d)$,

$$
R M S(d)=\sqrt{\frac{\sum_{j=1}^{k} d_{j}^{2}}{k}}
$$

Next, the obtained results are normalized,

$$
X_{\text {norm }}=\frac{X-X_{\min }}{X_{\max }-X_{\min }}
$$

The distance $d_{i}$ between the main cable point and the ideal point and the fitting precision $R M S(d)$ used to measure the main cable net surface and the ideal paraboloid are normalized. Then assign different weights to the two variables to realize the transformation from multi-objective to single-objective model, and make the optimization objective at this time as,

$$
\min Z=\varepsilon_{1} R M S(d)_{n o r m}+\varepsilon_{2} d_{\text {last_inorm }}
$$

Where, the weight $\left(\varepsilon_{1}, \varepsilon_{2}\right)$ is determined by our analysis as $\varepsilon_{1}=0.6832, \varepsilon_{2}=0.3168$.

\subsubsection{The Constraint}

Based on the above analysis, the determination of the ideal paraboloid is constrained by the following equations:

$$
\text { s.t. }\left\{\begin{array}{c}
x^{2}+y^{2}+z^{2}=R^{2} \\
x^{2}+y^{2}=2 p(z-a) \\
d_{j}=\sqrt{\left(x_{j P}-x_{j S P}\right)^{2}+\left(y_{j P}-y_{j S P}\right)^{2}+\left(z_{j P}-z_{j S P}\right)^{2}} \\
d_{\text {边 }}=\sqrt{\left(x_{B S}-x_{B S P}\right)^{2}+\left(y_{B P}-y_{B S P}\right)^{2}+\left(z_{B P}-z_{B S P}\right)^{2}} \\
n=\sum_{j=1}^{k} \operatorname{sgn} d_{j} \\
R M S d_{j}=\sum_{j=1}^{k} d_{j}
\end{array}\right.
$$

\subsection{Solution of the Model}

By using the ergodic search algorithm to solve, we can obtain the ideal parabola's focus point $P=140.3014$ and the bottom point $A^{\prime}(0,0,-300.715)$ of the parabola, then its equation can be obtained as,

$$
x^{2}+y^{2}=2^{*} 140.3014(z+300.715)
$$


Then, the image is drawn by using Matlab shown in figure 3, wherein, the green is the point that needs to be adjusted on the original reference paraboloid, and the black is the ideal paraboloid after adjustment,

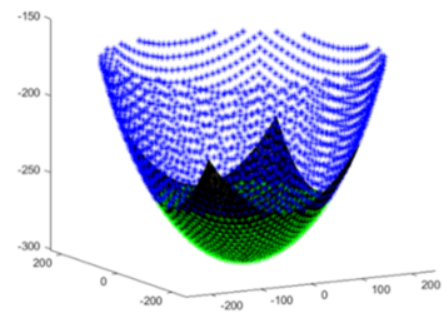

Figure 3. Schematic diagram of paraboloid

Next, we use the plane of symmetry to capture the difference between the ideal parabola and the reference parabola,the result is shown in figure 4,

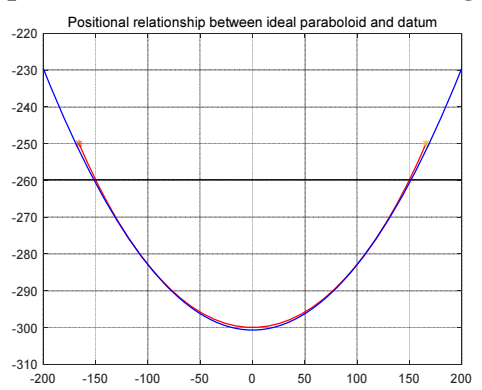

Figure 4. Comparison of ideal paraboloid and reference paraboloid

Among them, the red line in the figure represents the datum to be changed and the blue line represents the ideal paraboloid.

\section{Actual Working Face Adjustment}

\subsection{Euler Angular Coordinate Transformation}

Under the condition that the rotation angles of coordinate axes are known, $(\alpha, \beta):\left(0,90^{\circ}\right) \rightarrow\left(36.795^{\circ}, 78.169^{\circ}\right)$, the vertex invariant rotation transformation of the spatial rectangular coordinate system in the above problem is carried out by using Euler rotation transformation principle [1]. The result is shown in figure 5. 


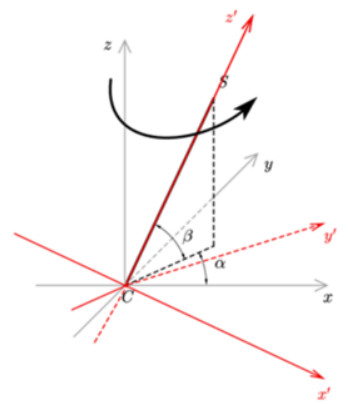

Figure 5. Transformation process of coordinate system

First, let the original coordinate axis rotate around the axis $z$, now $\theta_{3}=0$, then rotate around the axis $x$, and now $\theta_{1}=-a c \tan \left(\frac{\sin \alpha}{\tan \beta}\right)$, then rotate around the axis $y$. Finally, according to the above three rotations, the coordinate transformation formula in the new coordinate system can be obtained as,

$$
\left(\begin{array}{l}
x_{2} \\
y_{2} \\
z_{2}
\end{array}\right)=R_{y}\left(\theta_{2}\right) R_{x}\left(\theta_{1}\right) R_{z}\left(\theta_{3}\right)\left(\begin{array}{l}
x_{1} \\
y_{1} \\
z_{1}
\end{array}\right)
$$

\subsection{Working Parabolic Fitting}

We can directly use the ideal paraboloid determination model established above to solve the problem and obtain the ideal paraboloid image. The result are shown in the figure 6 and figure 7.

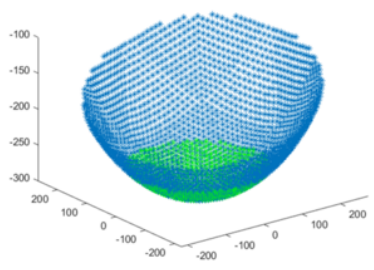

Figure 6. Side view of an ideal paraboloid

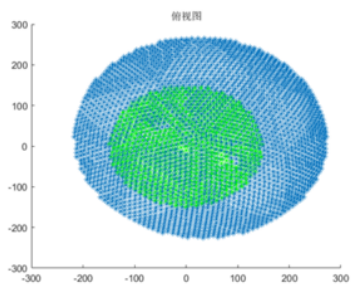

Figure 7. Top view of an ideal paraboloid

Layered iteration model through the analysis of the known data.The provisions of the benchmark of the location of the spherical vertex is the first layer, and according to the direction $z$ of the absolute value of the coordinates of the gradual decline in the sort of combination of gradually increase each layer corresponding to the main point number transformation rule, dividing layer[2].The result is shown in the figure 8. 

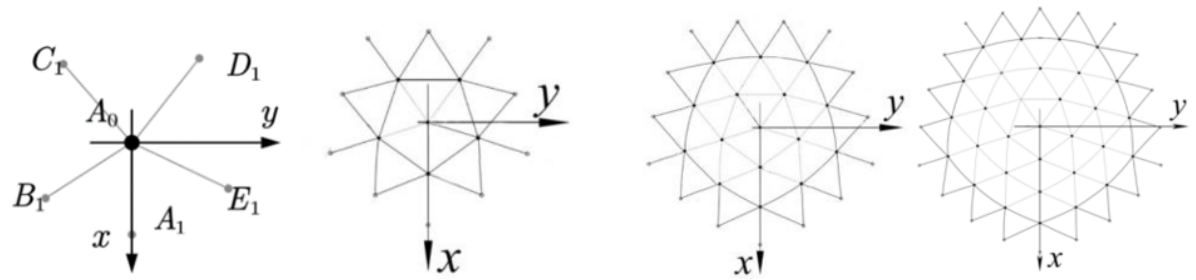

Figure 8. Reflection panel layer by layer iteration process

Next, we take layer as the research unit, and divide the reflector adjustment process into three processes: movement-judge-correction.

(1) The process of movement

In the process of layer - by - layer movement, it is necessary to determine the direction and distance of movement. In this process, we first set the displacement ratio $l$ of the main cable point, and made use of the principle of equal unit distance, that is,

$$
\frac{\left(x_{i}-x_{i-1}, y_{i}-y_{i-1}, z_{i}-z_{i-1}\right)}{l}=\frac{\left(x_{i 0}, y_{i 0}, z_{i 0}\right)}{L}
$$

Among it $L=\sqrt{{x_{i 0}}^{2}+y_{i 0}{ }^{2}+z_{i 0}{ }^{2}}$, and $\left(x_{i 0}, y_{i 0}, z_{i 0}\right)$ represents the initial position of the node in annex I corresponding to this layer. The scale adjustment is achieved and the initial adjustment position $\left(x_{i}, y_{i}, z_{i}\right)$ of the node is obtained.

(2) Judging process basis

The distance $d_{i 0}{ }^{\prime}, d_{i}{ }^{\prime}$ between $i$ 's adjacent nodes at the first layer before and after moving was calculated respectively,

$$
\begin{gathered}
d_{i}{ }^{\prime}=\sqrt{\left(x_{i 1}-x_{i 2}\right)^{2}+\left(y_{\mathrm{i} 1}-y_{i 2}\right)^{2}+\left(\mathrm{z}_{i 1}-\mathrm{z}_{i 2}\right)^{2}} \\
\Delta d_{i}=\frac{\left|d_{i}{ }^{\prime}-d_{i 0}{ }^{\prime}\right|}{d_{i 0}{ }^{\prime}} * 100 \%
\end{gathered}
$$

If $\Delta d_{i}<0.07 \%$, then it means that the moving process is true. If $\Delta d_{i}>0.07 \%$, then it indicates that the moving process is not valid, and the adjustment process needs to be amended.

(3) Correction process

The judgment of moving distance between adjacent layers and adjacent nodes is analyzed as in the above process. After judging the nodes of each layer after moving, the adjustment of nodes of each layer is revised.

\subsubsection{The Optimization Model}

We optimize the above moving-judge-correction reflector node adjustment process to make the final working paraboloid closer to the ideal paraboloid.

In the process of upward adjustment, after each change of the set $l$, the cycle adjustment of moving-judge-correction-move is carried out, and the calculation under the adjustment ratio $l$,

$$
R M S(d)=\sqrt{\frac{\sum_{i=1}^{k} d_{i}{ }^{2}}{k}}
$$




$$
\min _{l} R M S(d)
$$

Minimum is the objective function, gradually optimize the working parabola in the adjusting the ration $l$ process, until the fitting accuracy $R M S(d)$ between the working parabola and the ideal parabola converges to 0 , the cycle ends, and the optimal working parabola equation is obtained by calculating the corresponding optimal adjustment ratio $l^{*}$.

\subsection{The Error Analysis}

We analyze the radial distance error of 706 main cable points and the corresponding points of the ideal paraboloid,

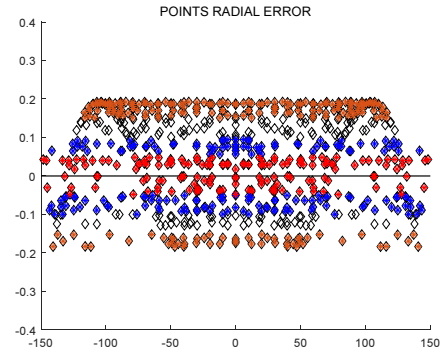

Figure 9. Radial distance error before iteration

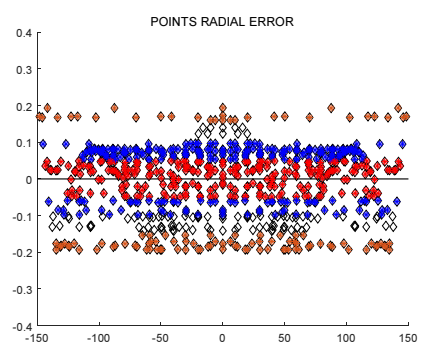

Figure 10. Radial distance error after iteration

In the figure 9 and figure 10, the absolute error values gradually decrease until the absolute error values corresponding to red dots are controlled in the internal $[0,0.1]$.

According to the above figure, it can be found that the magnitude of radial error transforms with the axis $x$ coordinates of the main cable point, and under the definite absolute error, the radial distance error of the main cable point fluctuates up and down around the horizontal straight line with the error of 0 , and gradually approaches the horizontal axis with the decrease of the absolute error.

\section{Conclusion}

In this paper, by studying the mechanism of the influence of the actuator radial expansion and contraction on the shape regulation of the active reflective surface, we establish an optimization model concerning the ideal paraboloid determination and the working paraboloid regulation. The model we developed is characterized by the following.

1. Converting the multi-objective into a single-objective optimization model in the process of ideal paraboloid determination, and normalizing the determined multiobjectives separately before weighting them, which makes the influence of the two objectives on the results more reasonable and accurate.

2. In the adjustment process of the reflection panel, the study object is converted from each main rigging point into each layer composed of point classes with the same distance from the origin, and a layered iterative method is used to combine the distance 
limitation between the main rigging points for the overall linkage, making the whole process more simple and efficient, comprehensive and accurate..

Meanwhile, for further research, we believe that improvements can be made in the following aspects.

3. The nodes of the main cable network will also have some influence on the parabolic surface in the process of radial contraction, and this one influence factor can be added.

\section{Acknowledgement}

This project was supported by the Science and Technology onHigh Power Microwave Laboratory Fund Project (Grant No.6142605180304). The authors acknowledge Dr. Hongliang Liu of University of Xiangtan for participating in many valuable discussions.

\section{References}

[1] Zhu L M, Wu X P, Li J W, Wu X. Euler rotation transformation and dynamic equation of rectangular coordinate system [J]. Marine surveying and mapping, 2010,30 (03): 20-22 + 40 .

[2] Liu Y. Research on reflector support mechanism of large aperture radio telescope [D]. Shanghai Jiaotong University, 2014. 